\title{
Higher Rate of Hyperbilirubinemia and Arrythmia in COVID-19 Cases Receiving Combination Therapy Atazanavir / ritonavir vs. Lopinavir / ritonavir
}

\author{
Hossein Mazaherpour ${ }^{1}$, Masoomeh Sofian ${ }^{1}$, Elham Farahani ${ }^{1}$, Alireza Abdi ${ }^{2}$, Sakine \\ Mazaherpour $^{3}$, Mona Sadat Larijani ${ }^{4}$, and Amitis Ramezani ${ }^{5}$ \\ ${ }^{1}$ Arak University of Medical Sciences \\ ${ }^{2}$ Kermanshah University of Medical Sciences \\ ${ }^{3} 3$. Treatment Management of Social Security Organization of Khuzestan Province \\ ${ }^{4}$ Pasteur Institute of Iran \\ ${ }^{5}$ Affiliation not available
}

April 27, 2021

\begin{abstract}
Background: Many treatments for COVID-19 are currently under studying, such as combination therapies with hydroxychloroquine plus antiviral drugs. In this study, we compared the efficacy and side effects of two types of combination therapy including atazanavir / ritonavir $(\mathrm{ATV} / \mathrm{r})$ or lopinavir /ritonavir (LPV/r) plus hydroxychloroquine among COVID-19 patients. Methods: In a non-randomized clinical trial, 108 eligible patients with moderate and severe form of COVID-19 were divided into two groups. Each group consisted of 54 patients. One group received ATV/r plus hydroxychloroquine and the other group received hydroxychloroquine plus LPV/r. Then, the two groups were evaluated and compared for clinical symptoms, recovery rates and complications of treatment regimens. Results: The findings of this research showed a significant increase in bilirubin in $\mathrm{ATV} / \mathrm{r}$ receiving group compared to $\mathrm{LPV} / \mathrm{r}$ receivers $(\mathrm{p}<0.001)$. there was also a significant increase in arrhythmias in the $\mathrm{LPV} / \mathrm{r}$ group compared to ATV/r group during the treatment period $(\mathrm{p}=0.019)$. Other findings including length of hospital stay, outcome, and treatment complications were not statistically significant. Conclusions: There is not statistically significant difference between protease inhibitor drugs including ATV $/ \mathrm{r}$ and LPV/r in the treatment of COVID-19 regarding to progress and clinical outcomes. However, some side effects such as hyperbilirubinemia and arrhythmia was significantly different by application of atazanavir or lopinavir.
\end{abstract}

Hossein Mazaherpour ${ }^{1}$, Masoomeh Sofian ${ }^{1 *}$, Elham Farahani ${ }^{1}$, Alireza Abdi ${ }^{2}$, Sakine Mazaherpour3, Mona Sadat Larijani ${ }^{4}$, Amitis Ramezani ${ }^{4^{* *}}$

1. Arak University of Medical Sciences, Arak, Iran.

2. Kermanshah University of Medical Sciences, Kermanshah, Iran.

3. Treatment Management of Social Security Organization of Khuzestan Province, Mahshahr, Iran.

4. Clinical Research Department, Pasteur Institute of Iran, Tehran, Iran.

**Corresponding Author:Amitis Ramezani (M.D. \& Ph.D.)

No:69, Pasteur Ave, Clinical Research Department, Pasteur Institute of Iran, Tehran 1316943551, Iran. Tel/Fax: +98(21)64112812 E.mail: amitisramezani@hotmail.com

*Co-corresponding Author: Masoomeh Sofian (M.D. \& Ph.D.)

Infectious Disease Research Center (IDRC), Arak University of Medical Science, Arak, Iran 
E-mail: dr.sofian@arakmu.ac.ir

\title{
Acknowledgements
}

This study was supported by Arak University of Medical Sciences, Arak, Iran (Grant number: 3619).

We would like to thank the medical teams of Ayatollah Khansari hospital and Amir Al-Momenin hospital for nice cooperation. We also appreciate Ahmad Akhlaghi for contribution in English edition of the manuscript.

\section{Conflict of Interest Statement}

The authors declare that they have no competing interests. This study approved by Ethics Committee of Arak University of Medical Sciences (approval ID: IR-ARAKMU.REC.1399.006). The study protocol was also registered as IRCT202005217047485N1. Written informed consents were obtained from the patients or one of the first degree family members if the patient was unconscious.

Higher Rate of Hyperbilirubinemia and Arrythmia in COVID-19 Cases Receiving Combination Therapy Atazanavir /ritonavir vs. Lopinavir / ritonavir

\begin{abstract}
Background: Many treatments for COVID-19 are currently under studying, such as combination therapies with hydroxychloroquine plus antiviral drugs. In this study, we compared the efficacy and side effects of two types of combination therapy including atazanavir / ritonavir (ATV/r) or lopinavir / ritonavir (LPV/r) plus hydroxychloroquine among COVID-19 patients.Methods: In a non-randomized clinical trial, 108 eligible patients with moderate and severe form of COVID-19 were divided into two groups. Each group consisted of 54 patients. One group received $\mathrm{ATV} / \mathrm{r}$ plus hydroxychloroquine and the other group received hydroxychloroquine plus LPV/r. Then, the two groups were evaluated and compared for clinical symptoms, recovery rates and complications of treatment regimens.Results: The findings of this research showed a significant increase in bilirubin in ATV/r receiving group compared to $\mathrm{LPV} / \mathrm{r}$ receivers $(\mathrm{p}<0.001)$. there was also a significant increase in arrhythmias in the $\mathrm{LPV} / \mathrm{r}$ group compared to $\mathrm{ATV} / \mathrm{r}$ group during the treatment period $(\mathrm{p}=0.019)$. Other findings including length of hospital stay, outcome, and treatment complications were not statistically significant.Conclusions: There is not statistically significant difference between protease inhibitor drugs including $\mathrm{ATV} / \mathrm{r}$ and $\mathrm{LPV} / \mathrm{r}$ in the treatment of COVID-19 regarding to progress and clinical outcomes. However, some side effects such as hyperbilirubinemia and arrhythmia was significantly different by application of atazanavir or lopinavir.Keywords: COVID-19, Combination therapy, atazanavir /ritonavir, lopinavir /ritonavir, Hyperbilirubinemia, ArrythmiaList of Abbreviations: ALP: Alkaline phosphatase, ALT: Alanine aminotransaminase, AST: Aspartate amino transaminase, BS: Blood Sugar, CBC: Complete Blood Count, CPK: Creatinine Phosphokinase, CRP: C-Reactive Protein, ECG: Electrocardiogram, ESR: Erythrocyte Sedimentation Rate, GIB: Gastrointestinal Bleeding, Hb: Hemoglobin, INR: International normalized Ratio, LDH: Lactate Dehydrogenase, PAC: Premature Atrial Contraction, PTE: Pulmonary Thromboembolism, RBBB: Right Bundle Branch Block, SpO2: Saturation of peripheral oxygen, WBC: White Blood Cell.IntroductionCoronavirus Disease 2019 (COVID-19) began in late 2019 in Wuhan, China, that spread rapidly to many countries of the world $[1,2]$. This pandemic disease has infected more than 110 million people worldwide [2, 3], which stems from a single stranded RNA virus from betacorona virus with humans and animals host [4-6]. The average incubation period of the disease is between 4 and 5 days, but may last up to 14 days $[5,7]$. The disease can be mild and asymptomatic to severe with ARDS (Acute Respiratory Distress Syndrome) and death. According to the results of studies, about $81 \%$ of patients present moderate and mild forms whereas $14 \%$ and $5 \%$ have severe and threatening to fatal forms of the disease, respectively [8-10]. Symptoms including fever, cough and dyspnea have been found in about $70 \%$ whereas muscle pain and headache were seen in $36 \%$ and $34 \%$ respectively [11]. Several therapeutics have
\end{abstract}


been investigated to overcome COVID-19 disease, however, neither of them have been approved internationally yet. Among these drugs, protease inhibitors which are used for treatment of HIV-1, are applied in COVID-19 [12]. Protease inhibitors impair the virus replication by inhibition of protease. Lopinavir /ritonavir $(\mathrm{LPV} / \mathrm{r})$ is a protease inhibitor drug that inhibits the protease 3CLpro enzyme, which is one of the RNA polymerase-dependent proteases of the virus and is involved in virus replication [12]. Atazanavir/ritonavir $(\mathrm{ATV} / \mathrm{r})$ is another drug belonged to protease inhibitors family that has better tolerance and fewer side effects comparing to LPV/r. Moreover, LPV/r has been shown to have better pulmonary permeability [13]. COVID-19 causes a widespread inflammatory response in the body through the cytokine storm mediated by interleukin-6. Some symptoms of COVID-19 are the result of this inflammatory reaction. Evidence showed that some protease inhibitors such as atazanavir can reduce symptoms and disease severity to some extent by decreasing the release of interleukin-6 from primary monocytes [14, 15]. Protease inhibitor drugs cause a number of side effects. Common side effects of LPV/r include nausea, vomiting, diarrhea, hepatotoxicity, and prolonged Q-T on electrocardiogram (ECG), skin rash, hyperlipidemia, and abdominal pain. Furthermore, ATV/r can cause some side effects such as hyperbilirubinemia, rash, fever, nausea, vomiting, diarrhea, high cholesterol and cough. Hyperbilirubinemia is the most frequently adverse effect of ATV. ATV can cause a reversible, dose-dependent increase predominantly of unconjugated bilirubin [16]. In previous studies, hydroxychloroquine caused some complications such as hemolysis in patients with glucose-6-phosphate dehydrogenase (G6PD) deficiency, bone marrow suppression, cardiomyopathy, some abnormalities in ECG including prolonged PR, QRS and QT interval, and some new onset cardiac arrhythmias such as bundle branching block (BBB), atrioventricular block [17, 18]. On the other hand, the definite treatment of COVID-19 is still unknown and the role and effectiveness of combination therapy in this disease is a matter to be investigated. In the present study, we aimed at efficacy and side effects comparison of two combination therapies including atazanavir / ritonavir (ATV/r) or lopinavir / ritonavir (LPV/r) plus hydroxychloroquine among COVID-19 patients.

\section{Methods}

Study design The study was designed as a double-blind and non-randomized clinical trial in which patients and questioner were not aware of the type of the treatment. Patients that came to the hospital were examined by an infectious specialist from who those with moderate or severe COVID-19 disease enrolled in this study. Overall, 108 eligible patients with COVID-19 that admitted to Ayatollah Khansari hospital and Amir AlMomenin hospital in Arak city were investigated from May to October 2020. Patients were non-randomly divided into two groups $(\mathrm{n}=54)$. Inclusion criteria were patients with moderate to severe COVID-19 that hospitalized in the infectious and COVID wards of hospital beside the signing the informed consent. Exclusion criteria were the critical type of the disease that required ICU admission and intubation, mild type of the disease without need to hospitalization, and unwillingness to participate in this study. Moreover, discharge was based on all of the followings: (1) Improvement in clinical signs and symptoms based on physician's opinion, (2) Afebrile status for $72 \mathrm{hrs}$ without antipyretics, (3) Saturation of peripheral oxygen (SpO2) more than $93 \%$ in ambient air without supplemental oxygen. In the present study, moderate and severe COVID-19 were defined according to WHO guideline. Therefore, moderate form of COVID-19 defined as existence of primary symptoms of pneumonia (dyspnea, cough and fever) and $\mathrm{SpO} 2[?] 90 \%$ in ambient air and severe form defined as profound pneumonia and $\mathrm{SpO} 2<90 \%$. The diagnosis approach was based on WHO guideline, so that positive reverse transcriptase-polymerase chain reaction (RT-PCR) of nasopharyngeal and oropharyngeal secretions or clinical manifestations plus findings of chest CT scan were highly suggestive for COVID-19 [7, 11].Therapeutic regimensPatients of one group received hydroxychloroquine tablet $400 \mathrm{mg}$ single dose on the first day and LPV/r (200mg/50mg tablet) 2 tablets every 12 hours from the second day for at least 5-7 days. The other group received hydroxychloroquine $200 \mathrm{mg}$ every 12 hours plus ATV/r 1 tablet (300mg/100mg) daily for at least 5-7days. According to physician's judgment the treatment period was longer whenever needed. The duration of treatment was at least 7 days. In addition, more treatments were also recorded such as other antiviral drugs, corticosteroids, intravenous immunoglobulin (IVIG), vitamin C, 
antibiotics, analgesic agents, anti-nausea and vomiting agents, cardiovascular drugs, deep vein thrombosis (DVT) and stress ulcer prophylaxis.Laboratory testsDemographic data, clinical presentations, underlying diseases, drug history, laboratory and medical data were collected and documented. Clinical symptoms such as fever, sore throat, dyspnea, abdominal pain and clinical signs including SPO2, pulse rate, respiratory rate, blood pressure and temperature were recorded at the time of hospital admission. Vital signs and laboratory tests such as white blood cells (WBC), serum electrolytes, liver and kidney enzymes, inflammatory biomarkers consisting C-reactive protein (CRP) and erythrocyte sedimentation rate (ESR) were extracted from the Hospital Information System (HIS) and patient's file every day and recorded in questionnaire. During the hospitalization, the patients were examined daily for the presence or absence of signs and symptoms of COVID-19 disease, as well as for vital signs and daily laboratory tests, and other information which were collected through a questionnaire. The patient's ECG was taken daily or every other day upon the admission and during the hospitalization. They were then interpreted by a cardiologist for the presence or absence of arrhythmia who was not aware of the patient's medication.Statistical analysisAll statistical analysis was performed using SPSS version 23.0. P-value less than 0.05 considered as statistically significant. Descriptive data were calculated as frequency, frequency percentage and analytical statistics through Chi-square test.

\section{Results}

This study was performed on 108 patients with COVID-19 who were in two groups of 54 patients. $47.2 \%$ of patients were male and $52.8 \%$ were female. The highest numbers of patients were in the age range of 60-79 years $(42.6 \%)$. The most common symptoms in both groups were dry cough (67.6\%), myalgia (65\%), dyspnea $(60.2 \%)$ and the least common symptom was skin rash $(8.3 \%)$. On admission, $27.8 \%$ of patients had SPO2> $94 \%$, and $41.7 \%$ of them had SPO2 between $90-94 \%$ and $30.6 \%$ had SPO2 less than $90 \%$. There was no significant difference between the two groups for the findings of physical examination at the time of admission including fever, blood pressure, respiratory rate, SPO2 and heart rate. Based on the findings of physical examination and evaluation of patients during the treatment period (Table 1), the number of days with fever $(\mathrm{P}=1.00)$, number of days with hypoxia $(\mathrm{p}=0.343)$, number of days with cough $(\mathrm{p}=0.334)$ and myalgia $(\mathrm{p}=0.412)$, and also length of stay $(\mathrm{p}=0.479)$ were not significantly different between two groups. Other findings including the number of days with dyspnea $(\mathrm{p}=0.417)$, number of days with headache $(\mathrm{p}=0.647)$, anorexia $(\mathrm{p}=0.90)$, nausea and vomiting $(\mathrm{p}=1.00)$, diarrhea $(\mathrm{p}=0.315)$. sore throat $(\mathrm{p}=1.00)$, abdominal pain $(\mathrm{p}=0.69)$ and skin lesions $(\mathrm{p}=0.08)$ that assessed daily for all patients in the course of treatment were not significantly different between two groups. On the first day of hospitalization, laboratory tests such as complete blood count (CBC), ESR, CRP, liver enzymes, bilirubin, lipid profile, serum lactate dehydrogenase $(\mathrm{LDH})$ and creatinine phosphokinase (CPK), and International normalized ratio (INR) were checked and were repeated during hospitalization according to the physician's opinion and existing instructions. Since the each of treatment regimen can lead to changes in these tests, the trend of changes were categorized as unchanged, ascending and descending according to the patient's baseline test and the normal range for these tests (Table 2 ). There was not statistically significant difference between two groups related to values of white blood cell (WBC) $(\mathrm{p}=0.127)$, hemoglobin $(\mathrm{p}=0.554)$, platelets $(\mathrm{p}=0.906)$ and lymphocyte percentage $(\mathrm{p}=0.478)$ (Table 2 ). What is more, there was not statistically significant difference for trend of ESR, CRP, creatinine and liver enzymes (Table 2). Bilirubin was significantly higher in the group of patients receiving ATV/r than the group of patients that receiving $\mathrm{LPV} / \mathrm{r}(\mathrm{p}<0.001)$. Hyperbilirubinemia is one of the most common side effects of $\mathrm{ATV} / \mathrm{r}$, and it is less common for LPV/r. The difference between two groups was not significant for other laboratory findings such as lipid profile, blood sugar, CPK, LDH and INR. The rate of arrhythmia in $\mathrm{LPV} / \mathrm{r}$ group was significantly higher than the ATV/r group $(\mathrm{p}=0.019)$ (Table- 3$)$. In this study, 44 patients suffered arrhythmias during treatment period including 28 patients of the LPV/r group and 16 patients of the ATV/r group. The types of arrhythmias for these patients were sinus bradycardia $(n=10)$, prolonged Q-T $(n=9)$, sinus tachycardia $(n=7)$, first-degree block $(n=6)$, left bundle branch block $(\mathrm{RBBB})(\mathrm{n}=6)$, right bundle branch block (RBBB) $(n=3)$, premature ventricular contraction $(\mathrm{PVC})(\mathrm{n}=2)$, and premature atrial contraction $(\mathrm{PAC})(\mathrm{n}=1)$. These arrhythmias were diagnosed by a cardiologist through comparing the initial 
ECG in admission day ECG of hospitalization days. In this research, treatment outcomes were classified as discharge with a good and stable general condition, evidence of complications during the hospital stay that continues until discharge or mortality. There is no significant difference in treatment outcomes between two groups. In this study, 5 patients expired; 3 patients in LPV $/ \mathrm{r}$ group and 2 patients in ATV/r group. Of these 5 patients, 4 were female and one was male. All 5 patients had SPO2 less than $90 \%$ on admission, two patients were over 80 years old and the other 3 patients were in the age range of $60-79$ years. One of these patients had no history of specific underlying disease, 2 patients had history of diabetes and hypertension, one patient had a history of hypertension and coronary heart disease and one patient had only a history of diabetes. 10 patients of $\mathrm{LPV} / \mathrm{r}$ group had complications during hospitalization and discharge. 4 patients had no increase in SPO2 and were dependent to oxygen on discharge, 2 patients had DVT, one patient had pulmonary thromboembolism (PTE), 2 patients had pressure ulcer, and one patient developed hemoptysis. In ATV/r group, 7 patients developed complications during hospitalization and on discharge, 3 patients did not increase SPO2 during hospitalization and on discharge, one patient developed pancreatitis, one patient had hemoptysis and one gastrointestinal bleeding (GIB).

\section{Discussion}

To the best of our knowledge, in few studies outcomes and complications of combination therapy were compared among COVID-19 hospitalized patients. In our clinical trial, the efficacy and side effects of two combination therapies with $\mathrm{LPV} / \mathrm{r}$ and $\mathrm{ATV} / \mathrm{r}$ plus hydroxychloroquine were compared between the two groups of COVID-19 patients. The results of this study indicated that this combination therapy with these drugs were not significantly different between two groups in term of hospital stay length, complications, mortality rate following treatment period, time of alleviation from clinical symptoms and clinical outcomes. The rate of hyperbilirubinemia was significantly higher in ATV/r group than LPV/r group. In this study, $85.4 \%$ of patients that received $\mathrm{ATV} / \mathrm{r}$ had hyperbilirubinemia. Atazanavir-induced hyperbilirubinemia is indirect hyperbilirubinemia that create by inhibition of the UDP-glucuronyl transferase enzyme. This enzyme conjugates bilirubin. Hyperbilirubinemia is usually mild and reversible [19]. The results of some studies revealed that boosted atazanavir with ritonavir increases the risk of indirect hyperbilirubinemia compared to atazanavir alone $[20,21]$. In a study by Malan et al which performed on 400 patients, the rate of hyperbilirubinemia was $59 \%$ in ATV/r group and $20 \%$ in atazanavir group [20]. The cardiac arrhythmias rate during treatment period was significantly higher in LPV/r group compared to ATV/r group. In this study, almost all the patients received antibiotic therapy in addition to antiviral therapy, and these antibiotics included fluoroquinolones and macrolides, which can cause arrhythmias for the patient. On the other hand, the interaction of LPV/r with these antibiotics, as well as with hydroxychloroquine, which has been used as part of combination therapy against COVID-19 in these patients, may be the cause of higher arrhythmias in the LPV/r group. The findings of Anson et al research showed that LPV/r could predispose patients to arrhythmias such as prolonged Q-T and torsade de point by inhibiting of human ether-a-go-go-related gene (HERG) potassium channels and potassium current (IKr) channels [22]. In Bessiere et al. study, combination therapy with hydroxychloroquine and azithromycin caused prolonged Q-T in 10.7-36\% of patients [23]. Russo et al study showed that $23 \%$ of patients reached developed arrhythmias following the treatment of COVID-19 with hydroxychloroquine and azithromycin [24]. The results of Moyle G et al. study, revealed a meaningful decrease in unconjugated and total bilirubin following zinc sulfate (ZnSO4) intake and a limited decrease in $\mathrm{ATV} / \mathrm{r}$ plasma exposure. ZnSO4 supplementation may represent a useful drug in the shortterm management of ATV- related hyperbilirubinemia in HIV-infected patients [25]. This finding disagrees with our research, that is related to significant decreasing effect of $\mathrm{ZnSO} 4$ on unconjugated bilirubin levels by inhibiting the enterohepatic cycling of unconjugated bilirubin. We did not assess the side effects and outcomes of combination therapies in different severity level of COVID-19 which was the limitation of the current study. Therefore, we recommend these items evaluation in future studies. 


\section{Conclusions}

In this study, two different combination therapy (ATV/r and LPV/r group) in term of efficacy and side effects were comparatively investigated. The findings indicated a significant increase in bilirubin in ATV/r receivers compared to $\mathrm{LPV} / \mathrm{r}$ treated patients. A significant increase in arrhythmias in the $\mathrm{LPV} / \mathrm{r}$ group compared to $\mathrm{ATV} / \mathrm{r}$ group was also seen during the treatment. The results of the current research indicated that apart from the rate of hyperbilirubinemia and cardiac arrhythmia, other indices were not statistically different between two groups. There was no significant difference for mortality rate and complications of treatment regimen between two groups. It seems that the current applying agents are not preferably acceptable to overcome COVID-19 infection and therefore more therapeutic regimens must be studied in this era.References:

1. Coronavirus disease 2019 (COVID-19): cases in U.S. [Internet]. Centers for Disease Control and Prevention. 2020 [cited 2020/04/09]. Available from: https://www.cdc.gov/coronavirus/2019-ncov/cases-updates/ cases-inus.html.

2. World Health Organization. [Internet]. World Health Organization. 2020 [cited 2020/06/09]. Available from: https://www.who.int/emergencies/diseases coronavirus-2019/novel- /situation reports/.

3. Rahmani H, Davoudi-Monfared E, Nourian A, et al. Comparing outcomes of hospitalized patients with moderate and severe COVID-19 following treatment with hydroxychloroquine plus atazanavir/ritonavir. DARU Journal of Pharmaceutical Sciences. 2020;28 (2):625-34.

4. Cui J, Li F, Shi Z-L. Origin and evolution of pathogenic coronaviruses. Nature Reviews Microbiology. 2019;17 (3):181-92.

5. Sofian M, Velayati AA, Banifazl M, et al. SARS-CoV-2, a virus with many faces: a series of cases with prolonged persistence of COVID-19 symptoms. Wiener Medizinische Wochenschrift. 2020.

6. Sadat SM, Aghadadeghi MR, Yousefi M, et al. Bioinformatics Analysis of SARS-CoV-2 to Approach an Effective Vaccine Candidate Against COVID-19. Molecular Biotechnology. 2021.

7. Guan W-j, Ni Z-y, Hu Y, et al. Clinical Characteristics of Coronavirus Disease 2019 in China. New England Journal of Medicine. 2020;382 (18):1708-20.

8. Salehi-Vaziri M, Jalali T, Farahmand B, et al. Clinical characteristics of SARS-CoV-2 by re-infection vs. reactivation: a case series from Iran. European journal of clinical microbiology \& infectious diseases : official publication of the European Society of Clinical Microbiology. 2021.

9. Wu Z, McGoogan JM. Characteristics of and Important Lessons From the Coronavirus Disease 2019 (COVID-19) Outbreak in China: Summary of a Report of 72314 Cases From the Chinese Center for Disease Control and Prevention. JAMA. 2020;323 (13):1239-42.

10. Fotouhi F, Salehi-Vaziri M, Farahmand B, et al. Prolonged Viral Shedding and Antibody Persistence in Patients with COVID-19. Microbes Infect. 2021:104810.

11. Stokes EK ZL, Anderson KN, et al. Coronavirus Disease 2019 Case Surveillance - United States January 22-May 30, 2020. MMWR Morb Mortal Wkly Rep. 2020.

12. Liu X, Wang X-J. Potential inhibitors against 2019-nCoV coronavirus M protease from clinically approved medicines. J Genet Genomics. 2020;47 (2):119-21.

13. Song S, Ji Y, Zhang G, et al. Protective Effect of Atazanavir Sulphate Against Pulmonary Fibrosis In Vivo and In Vitro. Basic \& Clinical Pharmacology \& Toxicology. 2018;122 (2):199-207.

14. Zhou F, Yu T, Du R, et al. Clinical course and risk factors for mortality of adult inpatients with COVID-19 in Wuhan, China: a retrospective cohort study. The Lancet. 2020;395 (10229):1054-62. 
15. Monteerarat Y, Sakabe S, Ngamurulert S, et al. Induction of TNF- $\alpha$ in human macrophages by avian and human influenza viruses. Archives of Virology. 2010;155 (8):1273-9.

16. Bissio E, Lopardo GD. Incidence of hyperbilirubinemia and jaundice due to atazanavir in a cohort of Hispanic patients. AIDS research and human retroviruses. 2013;29 (3):415-7.

17. Rathbun CR, Liedtke MD, Blevins SM, et al. Electrocardiogram Abnormalities with Atazanavir and Lopinavir/Ritonavir. HIV Clinical Trials. 2009;10 (5):328-36.

18. Joyce E, Fabre A, Mahon N. Hydroxychloroquine cardiotoxicity presenting as a rapidly evolving biventricular cardiomyopathy: key diagnostic features and literature review. Eur Heart J Acute Cardiovasc Care. $2013 ; 2$ (1):77-83.

19. Bentué-Ferrer D, Arvieux C, Tribut O, et al. Clinical pharmacology, efficacy and safety of atazanavir: a review. Expert Opin Drug Metab Toxicol. 2009;5 (11):1455-68.

20. Malan DR, Krantz E, David N, et al. Efficacy and safety of atazanavir, with or without ritonavir, as part of once-daily highly active antiretroviral therapy regimens in antiretroviral-naive patients. Journal of acquired immune deficiency syndromes (1999). 2008;47 (2):161-7.

21. Torti C, Lapadula G, Antinori A, et al. Hyperbilirubinemia during Atazanavir Treatment in 2,404 Patients in the Italian Atazanavir Expanded Access Program and MASTER Cohorts. Infection. 2009;37 (3):244.

22. Anson BD, Weaver JGR, Ackerman MJ, et al. Blockade of HERG channels by HIV protease inhibitors. The Lancet. 2005;365 (9460):682-6.

23. Bessière F, Roccia H, Delinière A, et al. Assessment of QT Intervals in a Case Series of Patients With Coronavirus Disease 2019 (COVID-19) Infection Treated With Hydroxychloroquine Alone or in Combination With Azithromycin in an Intensive Care Unit. JAMA Cardiol. 2020;5 (9):1067-9.

24. Russo V CA, Mottola FF, Mocerino R, Verde R, Attena E, Verde N, Di Micco P, Nunziata L, Santelli F, Nigro G, Severino S. Effect of Triple Combination Therapy With Lopinavir-Ritonavir, Azithromycin, and Hydroxychloroquine on QT Interval and Arrhythmic Risk in Hospitalized COVID-19 Patients. Front Pharmacol. 2020;11 (582348):1-5.

25. Moyle G, Else L, Jackson A, et al. Coadministration of atazanavir-ritonavir and zinc sulfate: impact on hyperbilirubinemia and pharmacokinetics. Antimicrobial agents and chemotherapy. 2013;57 (8):3640-4.

Table 1. Comparison of clinical presentations in COVID-19 patients on two different combination therapies.

\begin{tabular}{lllll}
\hline p-value & $\begin{array}{l}\text { Atazanavir/ritonavir } \\
\text { (percentage) }\end{array}$ & $\begin{array}{l}\text { Lopinavir/ritonavir } \\
\text { (percentage) }\end{array}$ & $\begin{array}{l}\text { Duration } \\
\text { (days) }\end{array}$ & \\
0.00 & $53(50)$ & $53(50)$ & $0-3$ & Febrile \\
0.479 & $1(50)$ & $1(50)$ & $3-5$ & \\
& $1(100)$ & $0(0)$ & $3-5$ & Admission \\
& $8(57.1)$ & $6(42.9)$ & $5-7$ & \\
& $20(46.5)$ & $23(53.5)$ & $7-9$ & \\
& $16(43.2)$ & $21(56.8)$ & $9-11$ & \\
& $8(66.7)$ & $4(33.3)$ & $11-13$ & \\
0.343 & $1(100)$ & $0(0)$ & $>14$ & \\
& $30(56.6)$ & $23(43.4)$ & $0-3$ & \\
& $11(37.9)$ & $18(62.1)$ & $3-5$ & \\
& $4(80)$ & $1(20)$ & $5-7$ & \\
& $2(66.7)$ & $1(33.3)$ & $7-9$ & \\
& $5(38.5)$ & $8(61.5)$ & $9-11$ & \\
& $2(40)$ & $3(60)$ & $11-13$ & \\
& & & &
\end{tabular}




\begin{tabular}{lllll}
0.412 & $44(47.8)$ & $48(52.2)$ & $0-5$ & Myalgia \\
& $9(60)$ & $6(40)$ & $5-10$ & \\
0.334 & $1(100)$ & $0(0)$ & $>10$ & \\
& $43(52.4)$ & $39(47.6)$ & $0-5$ & Cough \\
& $10(40)$ & $15(60)$ & $5-10$ & \\
0.417 & $1(100)$ & $0(0)$ & $>10$ & \\
& $42(47.2)$ & $47(52.8)$ & $0-5$ & Dyspnea \\
& $11(64.7)$ & $6(35.3)$ & $5-10$ & \\
0.647 & $1(50)$ & $1(50)$ & $>10$ & \\
& $52(50.5)$ & $51(49.5)$ & $0-5$ & Headache \\
0.90 & $2(40)$ & $3(60)$ & $5-10$ & \\
& $50(49.5)$ & $51(50.5)$ & $0-5$ & Anorexia \\
1.00 & $3(60)$ & $2(40)$ & $5-10$ & \\
& $53(50)$ & $53(50)$ & $0-5$ & Nausea \\
0.315 & $54(50)$ & $1(50)$ & $5-10$ & \\
& $0(0)$ & $53(49.5)$ & $0-5$ & Diarrhea \\
1.00 & $53(50)$ & $1(100)$ & $5-10$ & \\
& $1(50)$ & $53(50)$ & $0-5$ & Sore throat \\
0.696 & $4(57.1)$ & $1(50)$ & $5-10$ & \\
0.082 & $7(77.8)$ & $3(42.9)$ & $0-5$ & Abdominal pain \\
\hline
\end{tabular}

Table 2. Comparison of laboratory findings between COVID-19 cases receiving two types of therapeutics.

\begin{tabular}{|c|c|c|c|c|}
\hline $\mathrm{p}$-value & $\begin{array}{l}\text { Atazanavir/Ritonavir } \\
\text { (percentage) }\end{array}$ & $\begin{array}{l}\text { Lopinavir/Ritonavir } \\
\text { (percentage) }\end{array}$ & Trend & Features \\
\hline \multirow[t]{3}{*}{0.127} & $34(44.7)$ & $42(55.3)$ & No change & WBC on admission \\
\hline & $15(57.7)$ & $11(42.3)$ & Increasing & \\
\hline & $5(83.3)$ & $1(16.7)$ & Decreasing & \\
\hline \multirow[t]{3}{*}{0.554} & $49(49)$ & $51(51)$ & No change & $\mathrm{Hb}$ \\
\hline & $1(100)$ & $0(0)$ & Increasing & \\
\hline & $4(51.7)$ & $3(42.9)$ & Decreasing & \\
\hline \multirow[t]{3}{*}{0.906} & $37(51.4)$ & $35(48.6)$ & No change & Platelet \\
\hline & $4(50)$ & $4(50)$ & Increasing & \\
\hline & $13(46.4)$ & $15(53.6)$ & Decreasing & \\
\hline \multirow[t]{3}{*}{0.478} & $31(45.6)$ & $37(54.4)$ & No change & Lymphocyte count \\
\hline & $26(57.9)$ & $16(41.2)$ & Increasing & \\
\hline & $1(50)$ & $1(50)$ & Decreasing & \\
\hline \multirow[t]{3}{*}{0.836} & $22(53.7)$ & $19(46.3)$ & No change & ESR \\
\hline & $31(47.7)$ & $34(52.3)$ & Increasing & \\
\hline & $1(50)$ & $1(50)$ & Decreasing & \\
\hline \multirow[t]{3}{*}{0.587} & $21(50)$ & $21(50)$ & No change & CRP \\
\hline & $30(48.4)$ & $32(51.6)$ & Increasing & \\
\hline & $3(75)$ & $1(25)$ & Decreasing & \\
\hline \multirow[t]{2}{*}{0.82} & 41(49.4) & $42(50.6)$ & No change & Creatinine \\
\hline & $13(52)$ & $12(48)$ & Increasing & \\
\hline \multirow[t]{3}{*}{0.978} & $37(50.7)$ & $36(49.3)$ & No change & AST \\
\hline & $16(48.5)$ & $17(51.5)$ & Increasing & \\
\hline & $1(50)$ & $1(50)$ & Decreasing & \\
\hline
\end{tabular}




\begin{tabular}{lllll}
0.423 & $36(46.2)$ & $42(53.8)$ & No change & ALT \\
& $16(59.3)$ & $11(40.7)$ & Increasing & \\
\multirow{2}{*}{0.579} & $2(66.7)$ & $1(33.3)$ & Decreasing & \\
& $50(51.5)$ & $47(48.5)$ & No change & ALP \\
& $3(33.3)$ & $6(66.7)$ & Increasing & \\
$<0.001$ & $1(50)$ & $1(50)$ & Decreasing & \\
& $13(21.7)$ & $47(78.3)$ & No change & Bilirubin \\
0.380 & $41(85.4)$ & $7(14.6)$ & Increasing & \\
& $42(52.5)$ & $38(47.5)$ & No change & Lipid profile \\
0.288 & $12(42.9)$ & $16(57.1)$ & Increasing & \\
& $36(46.8)$ & $41(53.2)$ & No change & BS \\
0.790 & $18(58.1)$ & $13(41.9)$ & Increasing & \\
& $37(50.7)$ & $36(49.3)$ & No change & CPK \\
& $15(46.9)$ & $17(53.1)$ & Increasing & \\
0.841 & $2(66.7)$ & $1(33.3)$ & Decreasing & \\
& $36(49.3)$ & $37(50.7)$ & No change & LDH \\
& $16(50)$ & $16(50)$ & Increasing & \\
0.139 & $2(66.7)$ & $1(33.3)$ & Decreasing & \\
& $45(47.4)$ & $50(52.6)$ & No change & INR \\
\hline
\end{tabular}

Table 3. The outcome of two different combination therapies among COVID-19 patients

\begin{tabular}{lllll}
\hline p-value & $\begin{array}{l}\text { Atazanavir/ritonavir } \\
\text { (percentage) }\end{array}$ & $\begin{array}{l}\text { Lopinavir/ritonavir } \\
\text { (percentage) }\end{array}$ & \\
0.019 & $16(36.4)$ & $28(63.6)$ & Arrhythmia \\
0.633 & $45(52.3)$ & $41(47.7)$ & Discharge & End of treatment \\
& $7(41.20$ & $10(58.8)$ & Complication & \\
& $2(40)$ & $3(60)$ & expired & \\
\hline
\end{tabular}

